Umwelt und Entwicklung, Rio : Fünf Jahre danach

\title{
Handel und verkehr mit Kulturgütern stand der Regelung in der Schweiz
}

Catherine Schümperli Younossian

\section{(2) OpenEdition \\ 1 Journals}

Electronic version

URL: http://journals.openedition.org/sjep/759

DOI: 10.4000/sjep.759

ISSN: 1663-9677

Publisher

Institut de hautes études internationales et du développement

Printed version

Date of publication: 1 mars 1997

Number of pages: $277-288$

ISSN: 1660-5926

\section{Electronic reference}

Catherine Schümperli Younossian, « Handel und verkehr mit Kulturgütern stand der Regelung in der Schweiz », Schweizerisches Jahrbuch für Entwicklungspolitik [Online], 16 | 1997, Online erschienen am: 14 August 2012, abgerufen am 08 September 2020. URL : http://journals.openedition.org/sjep/759 ; DOI : https://doi.org/10.4000/sjep.759 


\title{
HANDEL UND VERKEHR MIT KULTURGÜTERN STAND DER REGELUNG IN DER SCHWEIZ
}

\author{
CATHERINE SCHÜMPERLI YOUNOSSIAN
}

$\mathrm{D}$

er internationale Handel mit Kulturgütern aller Art (Gemälde, Skulpturen, Antiquitäten, archäologische Gegenstände, moderne Kunst usw.) hat in den letzten Jahrzehnten markant zugenommen. Bis zu den 60er Jahren bestimmten in erster Linie Sammler und Kunstliebhaber das Geschehen auf dem Kunstmarkt. Nach und nach jedoch wurden Kulturgüter von Spekulanten als Kapitalanlage benutzt und immer mehr als reine Ware betrachtet. Der Wohlstand der 80er und 90er Jahre begünstigte den Aufschwung des Kunstmarktes. Das wichtigste Auktionshaus der Welt, Sotheby's, steigerte beispielsweise seinen Umsatz von 382 Millionen Dollar im Geschäftsjahr 1979/80 auf 3,2 Milliarden Dollar 1989/90, was eine Zunahme von 800 Prozent bedeutet. Der wachsende Umsatz im Kunsthandel in den letzten zehn Jahren geht auch auf die Ausdehnung des illegalen Handels mit Kulturgütern zurück. Die Gewinne daraus dienen verschiedenen Mafiakreisen, dem Drogenhandel und ähnlichen Milieus als Mittel zur Geldwäscherei. In diesem Artikel sollen die Missbräuche des illegalen Handels sowie die politischen und rechtlichen Gegenmassnahmen analysiert werden.

\section{HISTORISCHES ZEUGNIS ODER HANDELSWARE?}

Die besondere Schwierigkeit im Zusammenhang mit dem Kunsthandel liegt darin, dass Kunst- und Kulturgüter anderen Handelswaren nicht ganz gleichgestellt werden können. Der geschichtliche Wert, die Möglichkeit zur kulturellen Identifikation, die Einzigartigkeit und Unvermehrbarkeit sowie weitere mit dem Verstand nicht erfassbare Gründe heben sie von anderen Handelsgütern ab. Diese Besonderheiten und der immaterielle Wert von Kulturgegenständen führten zahlreiche Staaten dazu, die Ausfuhr von Gegenständen, die sie zu ihrem kulturellen Erbe zählen, zu verbieten. ${ }^{1}$ Die Attraktion archäologischer Gegenstände kann sehr folgenschwer sein, wie die Arbeitsgruppe ${ }^{2}$ in ihrem Bericht unterstreicht : « Die für Kulturgüter steigenden Preise führen immer mehr zur Ausplünderung der kulturgüterreichen Mittelmeerländer, aber auch einzelner Länder Asiens, Mittelamerikas und der Dritten Welt durch das organisierte Verbrechen. Hier geht es nicht nur um die einzelnen, weltweites Echo auslösenden Kunstdiebstähle, sondern weit mehr um systematische Raubgrabungen in grossem Umfang. Durch die dabei angerichtete Zerstörung von Funden und Fundzusammenhängen wird ausserdem wichtiges historisches Quellenmaterial vernichtet und der wissenschaftlichen Forschung für immer entzogen ».

\footnotetext{
' Handel und Verkehr mit Kulturgütern, Texte und erläuternder Bericht des Eidgenössischen Departements des Innern, Bern, August 1993.

2 Im Januar 1990 beauftragte das Bundesamt für Kultur eine Arbeitsgruppe, sämtliche Fragen im Zusammenhang mit dem Kulturgüterhandel zu prüfen. Der Bericht « Kulturgüter in der Schweiz - Einfuhr, Ausfuhr und Handel. Nationale und internationale Tragweite; Fragestellung, Lösungen und Auswirkungen » wurde dem Bundesamt für Kultur im Januar 1991 vorgelegt. Das Zitat stammt aus S. 7 des Berichts.
} 
Seit dem Fall der Berliner Mauer wird das Kulturerbe der osteuropäischen Länder systematisch geplündert. So sind den Museen in Rumänien in drei Jahren 11'000 Kunstwerke abhanden gekommen. Dabei wurden 60 Einbrüche und bewaffnete Raubüberfälle begangen. In Russland sind nach Ermittlungen der Behörden 40 organisierte Netze im Handel mit russischen Kunstgütern tätig. In der Tschechischen Republik wurden 1992 schätzungsweise 20'000 Kunstwerke ins Ausland geschmuggelt. In Polen wurden über 1'100 Kirchen geplündert, 100 Prozent mehr als Anfang der 80er Jahre. ${ }^{3}$ Auch der Süden bleibt nicht verschont : Die Kulturgüter zahlreicher asiatischer, afrikanischer und lateinamerikanischer Länder fallen Plünderungen und illegalem Handel zum Opfer. Besonders schlimm ist die Lage in Mali, wo, so Samuel Sidibé, Archäologe und Direktor des Nationalmuseums in Bamako, ein wesentlicher Teil des malischen Kulturerbes nach Europa und in die Vereinigten Staaten abgeflossen ist. In allen Regionen Malis kam es zu systematischen Plünderungen ; mancherorts wurden 80 bis 90 Prozent der archäologischen Stätten ausgeplündert. ${ }^{4}$ Angesichts dieser Lage unterzeichneten die Vereinigten Staaten mit Mali (sowie mit Mexiko, Peru und Guatemala, die von solchen Missständen besonders betroffen sind) bilaterale Abkommen über die Rückgabe gestohlener Kulturgüter. Gemäss der berühmten Fachzeitschrift « Archaeology » gehen Experten davon aus, dass über 90 Prozent der archäologischen Objekte, die heute auf den Markt kommen, aus illegalen Grabungen stammen. ${ }^{5}$

In einem zur Unidroit-Konvention veröffentlichten Artikel betonte Pierre Lalive d'Epinay $^{6}$, dass « der Interessenkonflikt zwischen den - oft unfreiwilligen Exporteuren von Kulturgütern und den Importeuren bedrohliche Ausmasse angenommen hat, welche die internationale Eintracht und den internationalen Frieden gefährden. Allerdings darf nicht vereinfachend mit dem Gegensatz Nord-Süd, Reiche-Arme, Exporteure-Importeure usw. argumentiert werden. Mehrere Länder (das beste Beispiel ist zweifellos Italien) sind bereits Exporteure und Importeure von Kunst- oder anderen Kulturgütern, eine Entwicklung, die im Interesse des internationalen Kulturaustausches zu begrüssen ist (...) Dessenungeachtet bestehen heute de facto erhebliche und sehr deutlich empfundene Unterschiede(...) » (Übersetzung aus dem französischen Originaltext). Diese Aussage wird von den Organisationen der Entwicklungszusammenarbeit teilweise angefochten ; sie stellen fest, dass der Strom der Kulturgüter zwischen der Dritten Welt und den Industrieländern de facto nur einseitig verläuft.

\section{DIE ROLLE DER SCHWEIZ}

Die Schweiz spielt auf dem Kunstmarkt eine sehr wichtige Rolle. Weltweit belegt sie den vierten Platz nach Grossbritannien, den USA und Frankreich. Im Jahr 1995 importierte die Schweiz « Kunstgegenstände, Sammlungsstücke und Antiquitäten $»^{7}$ im Wert von 315 Millionen Dollar ; die Exporte beliefen sich auf 415 Millionen Dollar. Bei diesen Zahlen handelt es sich um amtliche Angaben,

\footnotetext{
3 INFO, Informationsblatt der Schweizerischen UNESCO-Kommission, August 1993.

4 in Illicit traffic in cultural property, Samuel Sibide, Ed. by Harrie Leyten, Amsterdam 1995, p.8.

5 in Archaeology, sept./oct. 1992, p. 67.

6 in « Une avancée du droit international : la Convention de Rome d'Unidroit sur les biens culturels volés ou illicitement exportés », Uniform Law Review - Revue de droit uniforme 1996-1, p.57.

7 Schweizerische Aussenhandelsstatistik, 1995.
} 
welche die illegalen Importe nicht erfassen. 1992 befasste sich ein Artikel der Zeitschrift Bilanz ${ }^{8}$ mit den Gewinnen aus dem illegalen Kunsthandel. Der geschätzte Umsatz im illegalen Handel mit Kunst weltweit variiert je nach Quelle von 1,3 Milliarden (London Art Loss Register) über 2,6 Milliarden (Schätzungen der amerikanischen Regierung) bis 9 zu Milliarden Schweizer Franken (das britische Magazin Trace). Der Kunstmarkt ist wenig transparent : Neben dem legalen, für den Verkehr der Kunstgüter in den öffentlichen Museen und Privatsammlungen unerlässlichen Handel existiert auch ein illegaler und unannehmbarer Handel. In den letzten Jahren wurden zahlreiche Missbräuche aufgedeckt, bei denen die Schwarzhändler die Schwachstellen der schweizerischen Gesetze perfekt auszunutzen wussten.

\section{LÜCKEN IM SCHWEIZER RECHT}

Die Anziehungskraft der Schweiz für den illegalen Kunsthandel erklärt sich aus der äusserst liberalen Gesetzgebung. In der Schweiz gibt es derzeit auf Bundesebene keine Gesetzesnormen über die Exportkontrolle der Kulturgüter. Die Importe in die Schweiz sind frei. Da die Kantone ja keine zollrechtliche Kompetenz besitzen, wurden auch keine kantonalen Vorschriften zur Importkontrolle von Kulturgütern erlassen. ${ }^{9}$

Ferner werden Kulturgüter im Schweizer Recht (Zivilgesetzbuch) bedauerlicherweise wie gewöhnliche Waren betrachtet. Derzeit existieren keine besonderen zollrechtlichen Bestimmungen betreffend die Ein- und Ausfuhr von Kulturgütern. Zudem geniesst nach schweizerischem Recht der gutgläubige Erwerber grösseren Schutz als die Diebstahlopfer. Der gutgläubige Erwerber muss im Fall einer Rückgabe selbst dann entschädigt werden, wenn es sich nachweislich um ein gestohlenes Objekt handelte. Ein Rückforderungsgesuch muss innerhalb von nur 5 Jahren gestellt werden. Nach Ablauf dieser Frist kann der gutgläubige Erwerber nicht mehr gerichtlich belangt werden. Die schweizerischen Steuervorschriften gewährleisten die Anonymität von Verkäufer und Käufer. Die laxen Gesetze begünstigen auch das Auktionariat. Verkehr und Zwischenlagerung von Kulturgütern in Zollfreilagern sind keinerlei Kontrolle unterworfen. Ein einfaches Transitdokument mit einer Angabe zur Ware (z.B. Holzgegenstände) reicht aus.

Mit dem Bundesgesetz über internationale Rechtshilfe in Strafsachen, wonach geplünderte Länder die Beschlagnahme und Rückführung von gestohlenen Kulturgütern beantragen können, verfügt die Schweiz eigentlich über eine wirksame Handhabe. Allerdings ist das Gesetz an eine doppelte Voraussetzung geknüpft : Der im Rechtshilfegesuch geschilderte Sachverhalt muss dem Tatbestand einer nach Schweizer Recht verfolgten Straftat entsprechen. Wie schwer das Gesetz anwendbar ist, wird klar, wenn man bedenkt, dass sich für gestohlene archäologische Objekte aus illegalen Grabungen Beweisprobleme ergeben können. Trotzdem darf nicht vergessen werden, dass eine steigende Zahl von Rechtshilfegesuchen in Strafsachen um gestohlene oder illegal ausgeführte Kulturgüter an die

8 in Bilanz 6/1992, Geklaut wird auf Bestellung. S. 49.

9 Schlussbericht des Informationstages « Kulturgüter zwischen Markt und Museum », Schweizerische UNESCO-Kommission, Bern, 1993, S.42-44. 
Schweiz gerichtet werden und dass verschiedene Gerichtsurteile die Rückführung von Objekten, welche die Schweiz passierten, in das Ursprungsland fordern.

Im Gegensatz zu den Nachbarländern kennt die Schweiz demnach weder spezielle Vorschriften noch Import- oder Exportkontrollen für Kulturgüter. Angesichts dieses rechtsleeren Raumes schreibt die Arbeitsgruppe in ihrem Bericht : Es liegt « auf der Hand, dass sich unser Land zu einer internationale Drehscheibe für den Transit und den Handel mit illegalem Kunst- und Kulturgut entwickelt hat ${ }^{10}$. Nach der Verabschiedung der Richtlinie «Rückgabe» und der Verordnung " Ausfuhr » bezüglich der Kulturgüter in den EU- und EWR-Mitgliedstaaten dürfte sich die Anziehungskraft der Schweiz noch verstärken. Zudem haben die Vereinigten Staaten, Kanada und 80 weitere Staaten die UNESCO-Konvention von 1970 unterzeichnet. Im Juli 1995 erklärte Ridha Fraoua, Bundesamt für Justiz, in einem Interview mit einer welschschweizerischen Zeitung ${ }^{11}$ folgendes : « Für Händler und private Sammler wird die Schweiz ein noch interessanterer Handelsplatz für illegale Geschäfte(...). Es steht ausser Zweifel, dass die aktuelle Rechtslage der Schweiz den zunehmenden Missbräuchen Vorschub leistet. Eine politische Diskussion zu diesem emotionsgeladenen Thema ist daher wünschenswert. Da der Kulturgüterschutz Sache der Kantone ist, fehlen Angaben zur Gesamtzahl der in der Schweiz gestohlenen Kunstwerke. Allerdings weiss man, dass es weit mehr Diebstähle gibt als Prozesse » (Übersetzung aus dem französischen Originaltext).

Die Schlussfolgerungen des Berichts der Arbeitsgruppe (Januar 1991) schlugen zwei Abhilfemassnahmen vor :

- Schaffung einer - bis heute nicht existierenden - Verfassungsgrundlage für eine Regelungskompetenz des Bundes, damit der illegale Kulturgüterhandel wirksam bekämpft werden kann.

- Die Ratifikation der UNESCO-Konvention von 1970 über Massnahmen zum Verbot und zur Verhütung der unzulässigen Einfuhr, Ausfuhr und Übereignung von Kulturgut.

Mittlerweile hat sich das Völkerrecht mit der Unterzeichnung der Unidroit-Konvention über gestohlene oder rechtswidrig ausgeführte Kulturgüter vom 24. Juni 1995 in Rom weiterentwickelt.

« Aus Sorge über die beunruhigende Zunahme von Missbräuchen im internationalen Kulturgütertransfer ${ }^{12}$ hatte der Bundesrat zwei Vernehmlassungen bei den interessierten Kreisen durchgeführt. Die erste Vernehmlassung vom September 1993 bezog sich auf die beiden Vorschläge der Arbeitsgruppe (siehe oben). In der zweiten Vernehmlassung vom Januar 1996 wurden die betroffenen Kreise um Stellungnahmen zur Ratifikation der Unidroit-Konvention gebeten.

\footnotetext{
${ }^{10}$ Kulturgüter in der Schweiz - Einfuhr, Ausfuhr, Handel. Nationale und internationale Tragweite ; Fragestellung, Lösungen und Auswirkungen. Bericht der Arbeitsgruppe zu Handen des Bundesamtes für Kultur und des Eidgenössischen Departements des Innern. Januar 1991, S. 9.

${ }_{11}^{11}$ La Liberté, 18. Juli 1995, La restitution des oeuvres d'art volées sera réglementée.

${ }^{12}$ Begleitschreiben von Bundesrätin Ruth Dreifuss vom 29. Februar 1996 zum Vernehmlassungsverfahren über die Unidroit-Konvention.
} 
Im Jahr 1960 haben Peru und Mexiko im Rahmen der UNESCO Massnahmen zur Bekämpfung des illegalen Kunsthandels gefordert. Nachdem ein 1964 eingesetztes Expertengremium im August 1969 einen ersten Entwurf vorgelegt hatte, konnte die entsprechende UNESCO-Konvention am 14. November 1970 unterzeichnet werden.

Die Konvention verlangt die Rückgabe von gestohlenem oder rechtswidrig ausgeführten Kunstgut. Sie ist nicht rückwirkend und gilt nur für Objekte aus Museen und internationalen Einrichtungen. Die Vertragsstaaten verpflichten sich, die Ein- und Ausfuhr von Kulturgütern zu überwachen, bei der Erstellung von Inventaren zusammenzuarbeiten und sich gegenseitig über die Behandlung dieser Objekte zu informieren. Ferner verpflichten sie sich zu gegenseitiger Unterstützung bei der Aufdeckung illegaler Geschäfte. Die UNESCO-Konvention wurde bereits von 82 Staaten ratifiziert, darunter zahlreiche europäische Länder, die USA und Kanada. Sie ist als Staatsvertrag nicht direkt anwendbar (nicht self-executing) - es bedarf also der ausdrücklichen Umsetzung der Bestimmungen in das Landesrecht der Vertragsparteien. Die Konvention hat verschiedene Kritiken hervorgerufen. Die sehr offene Formulierung, insbesondere zur Definition der Kulturgüter, erlaube einen grossen Spielraum bei der Umsetzung ins Landesrecht. Bisweilen werden dem Übereinkommen auch eine Tendenz zum kulturellen Nationalismus und eine zu einseitige Berücksichtigung der Interessen vorgeworfen. Daraus erklärt sich der geringe Erfolg der Konvention bei den « kulturimportierenden » Nationen. Zudem verfügt die Konvention über einen sehr eingeschränkten Geltungsbereich, der nur die Rückgabe von in Museen oder ähnlichen Einrichtungen gestohlenen Kulturgütern umfasst. Im Bewusstsein um ihre Grenzen hat die UNESCO im übrigen Unidroit aufgefordert, sich dieser Frage anzunehmen.

Im September 1993 eröffnete der Bundesrat bei den interessierten Kreisen eine Vernehmlassung zur Ratifikation der UNESCO-Konvention. Die im Januar 1996 veröffentlichten Vernehmlassungsergebnisse lassen ersehen, dass eine überwiegende Mehrheit der Befragten (75\%) dem Bund eine neue Gesetzgebungskompetenz im internationalen Kulturgütertransfer einräumen möchte, die Ratifikation der UNESCO-Konvention bejaht und die Erarbeitung eines entsprechenden Bundesgesetzes befürwortet. Die beiden Pole in der Debatte bilden die Akteure im Kunsthandel, welche eine Regelung vehement ablehnen, und die Entwicklungsorganisationen. Die Kreise des Kunsthandels schliessen jegliche Gefahr für die Ausplünderung von Kulturressourcen der armen Länder aus ; nach ihrer Meinung ist der Kulturaustausch unerlässlich und für alle vorteilhaft. Die Organisationen der Entwicklungszusammenarbeit ihrerseits halten Massnahmen zur Bekämpfung der Missbräuche und des illegalen Verkehr für dringend notwendig. Vor diesem Hintergrund würde die Ratifikation der Konvention - trotz deren Schwächen - ein politisches Signal setzen und die Bereitschaft zur Zusammenarbeit bei der Bekämpfung der illegalen Tätigkeiten zu bekunden. Zur Mehrheit der Befürworter einer Regelung gehören die meisten kulturellen Organisationen, ferner sämtliche schweizerischen Museenverbände, die politischen Parteien, die Kantone und verschiedene andere Bewegungen (Organisationen der Entwicklungszusammenarbeit, Frauen- und kirchliche Organisationen). Einzig die Organisationen des Kunsthandels, der Vorort, der SGV, die Kantone Genf und Waadt, 
die SVP und die LPS stehen der Ratifikation negativ gegenüber. Im Presserohstoff des Eidgenössischen Departements des Innern vom Januar 1996 ist dazu zu lesen : « Der Bundesrat wertet daher das Resultat der Vernehmlassung als Aufforderung, in dieser Materie gesetzgeberisch tätig zu werden, weil Handlungsbedarf sowohl aus kulturpolitischer wie auch aussenpolitischer Sicht besteht ${ }^{13}$.

\section{UNIDROIT-KONVENTION}

Der Text der Unidroit-Konvention über gestohlene oder rechtswidrig ausgeführte Kulturgüter vom 24. Juni 1995 ist das Resultat zehnjähriger Arbeiten, an denen sich zwei internationale Organisationen - die UNESCO und Unidroit (Internationales Institut für die Vereinheitlichung des Privatrechts) - sowie eine grosse Anzahl Experten beteiligt haben. Die Schweiz war von Anfang an vertreten. In den Kommissionen sowie in der diplomatischen Konferenz, die vom 7. bis 24. Juni 1995 in Rom stattfand, spielte sie eine prägende und vermittelnde Rolle. An der Konferenz nahmen 78 Staaten aus allen Kontinenten teil. Bis heute haben 22 Länder die Konvention unterzeichnet, darunter Italien, Frankreich, Finnland, die Niederlande und die Schweiz.

\section{Beispiele von Rückgabeklagen verschiedener Staaten}

Am 7. November wurden in der Galerie Koller rund 400 präkolumbische Objekte aus Peru, Bolivien, Mexiko und Guatemala versteigert. Der Hauptteil dieser Objekte kam aus Peru.

Die peruanische Botschaft ist vor der Versteigerung auf diese Auktion aufmerksam geworden und hat bei den zuständigen Stellen in Peru abklären lassen, ob es sich bei diesen Objekten um rechtmässig exportierte Stücke handelt. Die Abklärungen haben ergeben, dass die fraglichen Kulturgüter aus illegalen Ausgrabungen stam- men : In Peru müssen nämlich alle Kulturgüter, ob in Museums- oder Privatbesitz, registriert werden. Die zum Verkauf in Zürich angebotenen Stücke waren das nicht, müssen nach Ansicht des peruanischen Kulturministeriums daher illegal gegraben worden sein. Peru hat in den letzten 20 Jahren auch keine Exportbewilligung für ähnliche Stücke erteilt. Peru ist von illegalen Grabungen und der Plünderung seiner Kulturschätze besonders stark betroffen.

Peru gehört zu den lateinamerikanischen Staaten, die besonders stark von Raubgrabungen (und den Zerstörungen, die diese immer begleiten) betroffen sind. Die USA, die ja auch die Unesco-Konvention von 1970 ratifiziert haben, haben deshalb mit einigen dieser Staaten - Peru, Guatemala, Mexiko - bilaterale Zusatzabkommen abgeschlossen, die die Rückgabe von illegal exportiertem Kulturgut vorsehen.

Quelle :

Internet-Adresse der Erklärung von Bern : http ://www.access.ch/evb

Facts 46/1995. Heisse Ware unter dem Hammer

${ }^{13}$ Presserohstoff des EDI, Januar 1996, « Erläuterungen zur Ausgangslage UNESCO-Konvention 1970 und UnidroitKonvention ». 
Ausgehend von der Erkenntnis, dass nur eine internationale Konvention die Rechtsordnungen von rund 200 souveränen Staaten ansatzweise vereinheitlichen könne, formulierte ein Arbeitsausschuss unter dem Vorsitz von Professor Pierre A. Lalive d'Epinay von der Universität Genf einen Unidroit-Konventionsentwurf über Kulturgüter. Da der Kunsthandel von privaten Handlungen geprägt ist (Verkaufvertrag, Eigentumsübertragung an einen gutgläubigen Erwerber usw.) musste die Thematik auf privatrechtlicher Basis angegangen werden. Dabei galt es zu vermeiden, dass interessierte Milieus von den unterschiedlichen Gesetzen der verschiedenen Länder profitierten. Gleichzeitig sollten die wesentlichen Grundsatzerklärungen der UNESCO-Konvention auf wirksame Weise aufgegriffen werden.

Einige Punkte bereiteten den Experten Schwierigkeiten : die Abgrenzung der zu regelnden Bereiche, die Bestimmung des Begriffes « Kulturgut » und die grosse Vielfalt der nationalen Rechtsvorschriften und juristischen Traditionen.

Wie Professor Lalive d'Epinay betont ${ }^{14}$, "stellt die anhaltende Expansion des illegalen Handels und vor allem des Diebstahls von Kulturgütern einen echten Skandal dar, der auf effizienteste Weise bekämpft werden muss. Nicht nur Private, das Gemeinwesen, Sammler und Museen, sondern auch die Kulturgegenstände selbst werden beeinträchtigt (wie das Beispiel der archäologischen Raubgrabungen belegt) »(Übersetzung aus dem französischen Originaltext). Um diese Missstände zu beheben, verfolgt die Konvention zwei Zielsetzungen : in bezug auf gestohlene Kulturgüter (Kapitel II) und in bezug auf rechtswidrig ausgeführte Kulturgüter (Kapitel III). Diese Kapitel bilden eine Einheit, die als solche angenommen oder abgelehnt wird.

Die Unidroit-Konvention ist im Gegensatz zur UNESCO-Konvention direkt anwendbar (self-executing) ${ }^{15}$ - sie bedarf also keiner Umsetzung in das Landesrecht der Vertragsparteien. Zum ersten Mal wurden in einem normativen Akt mit allgemeiner Bedeutung die Bedingungen für die Rückgabe bzw. Rückführung von gestohlenen bzw. rechtswidrig ausgeführten Kulturgütern sowie der Mechanismus des Verfahrens auf Ansprüche internationalen Charakters festgelegt. Zwei Punkte sind besonders hervorzuheben : erstens, dass die Konvention nicht rückwirkend und deshalb nicht auf Kulturgüter anwendbar ist, die vor ihrem Inkrafttreten gestohlen oder rechtswidrig ausgeführt worden sind; zweitens, dass sie eine angemessene Entschädigung für den gutgläubigen Erwerber vorsieht, der ein gestohlenes oder illegal ausgeführtes Kulturgut zurückzugeben hat. Das zweite Kapitel der Konvention erwähnt den Rückgabeanspruch für gestohlene oder rechtswidrig ausgegrabene Kulturgüter und präzisiert die Voraussetzungen für die Rückgabe der gestohlenen Objekte. Das dritte Kapitel sieht einen Rückforderungsanspruch für rechtswidrig ausgeführte Kulturgüter vor, die - unter Verlet-

\footnotetext{
${ }^{14}$ in « Une avancée du droit international : la Convention de Rome d'Unidroit sur les biens culturels volés ou illicitement exportés », Revue de droit uniforme 1996-1, p. 48.

15 « Self-executing »-Verträge sind in jedem Vertragsstaat unmittelbar anwendbar, ohne dass es interner Bestimmungen bedarf. Nach der Ratifikation und der Veröffentlichung werden sie zu einem Teil des innerstaatlichen Rechts : Sie sind damit als Rechtsquellen automatisch der Verfassung, dem Gesetz und den allgemeinen Bundesbeschlüssen oder Verordnungen gleichgestellt. Der Bundesrat kann sich zum Erlass der notwendigen Vorschriften auf einen « self-executing »-Vertrag stützen, wobei er im Rahmen von Verordnungen die Vertragsnormen lediglich konkretisiert, aber keine neuen schafft. Die Klauseln eines « self-executing »-Vertrags müssen so präzise sein, dass sie im Einzelfall direkt einer Lösung zugrunde gelegt werden können; (...) in André Grisel, Traité de droit administratif, vol. 1, éd. IDES et CALENDES, Neuenburg, 1994, S. 91 (Übersetzung aus dem französischen Originaltext).
} 
zung der Gesetzgebung eines Vertragsstaates, welche die Ausfuhr von Kulturgütern regelt - aus dem Territorium eines solchen verbracht wurden. Für die Rückführung der rechtswidrig ausgeführten Kulturgüter von bezeichnender Bedeutung in den klagenden Staat müssen bestimmte Voraussetzungen erfüllt sein.

Die Unidroit-Konvention ermöglicht es, gegen Missbräuche im internationalen Verkehr mit Kulturgütern anzukämpfen. Gleichzeitig gibt sie auch jedem rechtmässigen Eigentümer - seien es ein Staat, ein Museum oder privat Sammelnde einen juristischen Mechanismus in die Hand, dank welchem Ansprüche auf Rückforderung gestohlener oder rechtswidrig ausgeführter Kulturgüter bei einem ordentlichen Gericht geltend gemacht werden können. Im Fall von Rückgabeforderungen ausländischer Besitzer oder Staaten würden also schweizerische Gerichte und schweizerische Richter über die Rechtmässigkeit der Ansprüche entscheiden.

Im Januar 1996 ermächtigte der Bundesrat das Eidgenössische Departement des Innern, ein Vernehmlassungsverfahren über die Unidroit-Konvention durchzuführen, um die interessierten Kreise zu einer Stellungnahme zur Ratifikation der Konvention aufzufordern. Es handelt sich hier um eine Vorlage mit Fragen von grosser Tragweite für die künftige Politik auf dem Gebiet des internationalen Kulturgütertransfers der Schweiz. Zahlreiche Stellungnahmen, über 120, wurden abgegeben. Kantone, politische Parteien, Wirtschaftsverbände, Organisationen des Kunsthandels, kulturelle Organisationen und Organisationen der Entwicklungszusammenarbeit reichten dichte und ausführliche Kommentare zum Thema ein. Die überwiegende Mehrheit der in der Vernehmlassung Befragten begrüsste die Ratifikation der Konvention und unterstützte die darin vorgeschlagenen Mechanismen. 23 Kantone, die CVP, die SPS, der Schweizerische Gewerkschaftsbund, interkommunale und internationale Organisationen, eine breite Mehrheit der kulturellen Organisationen sowie die kirchlichen Organisationen, Frauenorganisationen und die Organisationen der Entwicklungszusammenarbeit befürworteten die Ratifikation. Gegen eine Ratifikation der Unidroit-Konvention sprachen sich die Kantone Bern und Genf, die FDP, die SVP, der Vorort, der Schweizerische Gewerbeverband sowie die Organisationen des Kunsthandels aus.

Die Befürworter der Konvention begrüssen es, dass der Bundesrat einen weiteren, konsequenten Schritt einleiten will, um die beunruhigende Zunahme von Missbräuchen im internationalen Kulturgütertransfer einzudämmen. Die Unidroit-Konvention sei ein griffiges Mittel zur Bekämpfung solcher Missbräuche. Ihre Ratifikation dränge sich aus ethischen, juristischen und kulturpolitischen Gründen auf. Ein Verzicht auf ihre Ratifikation würde wegen des Fehlens wirksamer Schutzmechanismen in der Schweiz die Sogwirkung für allfällige dubiose Geschäfte noch deutlich verstärken. Für die Gegner der Ratifikation schiesst die Konvention über das Ziel hinaus. Sie sei kein geeignetes Instrument, um den Ausgleich aller Interessen im internationalen Kulturgütertransfer zu gewährleisten. Ferner befürchten sie grosse Beeinträchtigungen für den Handel und das Sammeln.

Der Bundesrat hat vom Ergebnis der Vernehmlassung Kenntnis genommen. Da eine überwiegende Mehrheit der Vernehmlassenden die Ratifikation befürwortete, beschloss er, die Unidroit-Konvention ${ }^{16} \mathrm{zu}$ unterzeichnen, was am 30. Juni

${ }^{16}$ Für weitere Einzelheiten siehe die Ergebnisse des Vernehmlassungsverfahrens zur Unidroit-Konvention (beim EDI erhältlich). 
1996 in Rom geschah. Eine Arbeitsgruppe unter Federführung des EDI wurde beauftragt, die rechtlichen Fragen im Zusammenhang mit der UNESCO-Konvention von 1970 und der Unidroit-Konvention zu prüfen und die Folgearbeiten zu koordinieren.

\section{Illegal aus der Türkei exportierte Grabstelen im Basler Antikenmuseum - Türkei klagt auf Rückgabe}

Seit 1979 sind im Basler Antikenmuseum fünf phrygische Grabstelen ausgestellt, die in den 70er Jahren illegal aus der Türkei exportiert worden sind. Die Türkei klagt auf Rückgabe dieser Stelen und hat ein Urteil des Appellationsgerichtes Basel 1995 ans Bundesgericht weitergezogen.

Zwei der fraglichen Stelen waren 1973 vom deutschen Professor Thomas DrewBear im türkischen Dorf Gökceler gesichtet, fotografiert und gezeichnet worden. Nach Aussagen der Leute im Dorf waren sie kurz zuvor von Einheimischen ausgegraben worden. Prof. Drew meldete den Fund sofort den Behörden des kleinen Dorfs. In den späten 80er Jahren erkannte Drew-Bear die beiden Grabstelen auf Abbildungen in einer wissenschaftlichen Studie wieder. Eine der Stelen war unterdessen aus kommerziellen Gründen durchsägt worden. Die türkischen Behörden reagierten, nachdem sie davon Kenntnis erhalten hatten, 1990 mit einer Klage auf Rückgabe.

Das Appellationsgericht Basel hat die Zeugenaussage von Prof. Drew-Bear als glaubwürdig erachtet. Die Klage der Türkei wurde vom Appellationsgericht aber trotzdem abgelehnt. Illegaler Export ist kein Deliktbestand in der Schweiz. Dass Bodenfunde Eigentum des Staates seien, wurde zwar anerkannt, das Gericht argumentierte aber, die türkischen Behörden hätten es versäumt, die Stelen schnell zu registrieren. Angesichts der Tatsache, dass die Stelen in einem kleinen ländlichen Dorf lagen, ist das eine sehr fragwürdige Begründung. Der Fall wurde ans Bundesgericht weitergezogen. Ein Entscheid des Bundesgerichtes steht noch aus.

Die Unidroit-Konvention ist nicht rückwirkend. Wenn die Schweiz sie 1995 unterzeichnet hätte, hätte das an der Rechtssituation für diese Klage also nichts geändert. Bei illegalen Exporten, die nach der Ratifikation der Unidroit-Konvention erfolgen, wäre diese Konvention hingegen ein wirksames Instrument, um die Rechte des rechtmässigen Besitzers zu garantieren.

\section{Quelle:}

Internet-Adresse der Erklärung von Bern : http ://www.access.ch/evb

Basler Zeitung : 11.8.1995, Türkei fordert von Basel antike Grabsteine zurück

Basler Zeitung : 19.8.1995, Antike Grabsteine können in Basel bleiben

\section{SCHLUSSFOLGERUNG}

Die ungebrochene Zunahme des Handels mit gestohlenen Kulturgütern und des illegalen Verkehrs gibt Anlass zu Besorgnis. Um die Missstände zu bekämpfen, ist die internationale Zusammenarbeit von Museen, Kunsthändlern (welche ihre Berufsethik verstärken sollten), Verwaltung, Polizei, Versicherungswesen, Juristen usw. unerlässlich. Allgemein wird anerkannt, dass die Probleme nicht auf nationaler Ebene geregelt werden können. Einzig eine bessere internationale Zusammenarbeit bietet einen Lösungsansatz. Dass im Abstand von 25 Jahren 
zwei völkerrechtliche Konventionen zu diesem Thema erarbeitet wurden, zeugt von einem wachsenden Konsens unter den Nationen für eine Eingrenzung der Grauzonen im internationalen Kulturgütertransfer. Zudem ist angesichts der Schwierigkeiten zahlreicher « kulturexportierender » Staaten bei der Durchsetzung ihrer einschlägigen Gesetze die Zusammenarbeit der « kulturimportierenden » Länder aus politischen und aus ethisch-moralischen Gründen erforderlich, als Akt der internationalen Solidarität und zum Schutz des eigenen Kulturgutes.

Wie oben dargestellt weitet sich die Kluft zwischen dem europäischen Recht und der schweizerischen Rechtsordnung aus. Die Schweiz hat sich mit ihrer Anziehungskraft auf zahlreiche (darunter auch wenig skrupelhafte) Händler zu einer Drehscheibe im illegalen Kunsthandel entwickelt - eine Einschätzung, die durch die jüngsten Skandale leider nur bestätigt wird. Die Schweiz darf nicht länger tolerieren, dass ihr Territorium für den illegalen Kulturgütertransfer missbraucht wird. Die Ergebnisse der beiden unlängst bei den interessierten Kreisen durchgeführten Vernehmlassungen lassen den Willen ersehen, diese untragbare Situation zu beenden. Es ist dringend nötig, zu handeln. Dies hat auch der Bundesrat erkannt. Die Schweiz hat deshalb bei den Vorarbeiten und in der Unidroit-Konferenz in Rom eine sehr aktive Rolle gespielt und gehört zu den Erstunterzeichnern der Unidroit-Konvention. Wichtige Massnahmen sind bereits ergriffen worden ; nun müssen politische Entscheidungen - zur Ratifikation der UNESCOKonvention und zur Unidroit-Konvention - die Fortschritte untermauern. Wir sind zuversichtlich, dass die Schweiz die erforderlichen Massnahmen treffen wird, um ihre Glaubwürdigkeit bei der internationalen Staatengemeinschaft wiederherzustellen.

\section{Chronologie der wichtigsten Ereignisse}

Januar 1990 Aufgrund zunehmender Vorstösse aus dem Ausland und wegen Vorkommnissen im Inland setzte das Bundesamt für Kultur am 7. Januar 1990 eine Arbeitsgruppe zur Abklärung der Fragen im Zusammenhang mit der Ausfuhr schweizerischer und der Einfuhr ausländischer Kulturgüter ein.

Januar 1991 Der Bericht der Arbeitsgruppe «Kulturgüter in der Schweiz - Einfuhr, Ausfuhr, Handel. Nationale und internationale Tragweite ; Fragestellung, Lösungen und Auswirkungen » wird im Januar 1991 eingereicht. Er schlägt zwei Hauptmassnahmen vor :

- Ratifikation der UNESCO-Konvention über Massnahmen zum Verbot und zur Verhütung der unzulässigen Einfuhr, Ausfuhr und Übereignung von Kulturgut ;

- Schaffung einer Verfassungsgrundlage für eine Regelungskompetenz des Bundes bezüglich der Ein- und Ausfuhr von Kulturgütern.

Legislaturplanung 1991 - 1995

Im Bericht über die Legislaturplanung 1991 - 1995 vom 25. März 1992 
erklärte der Bundesrat : " Handlungsbedarf besteht auch im Bereich des Kulturgüterhandels. Hier verfügt die Schweiz im Gegensatz zu den meisten europäischen Staaten über keinerlei rechtliche Kontroll- und Steuerungsmöglichkeiten. Nicht ganz unbegründet sieht sich unser Land dem Vorwurf ausgesetzt, Umschlagplatz für den illegalen Handel mit ausländischen Kulturgütern zu sein » (BBI 1992 III 115). Der Bundesrat nahm in Aussicht, das Problem des Kulturgüterhandels anzugehen und die beiden Vorschläge der Arbeitsgruppe zu prüfen (BBl 1992 III 116).

April 1992 Der Bericht wird über ein Jahr nach seinem Erscheinen veröffentlicht.

Neuerscheinung bei der Erklärung von Bern: «Götter, Gräber und Geschäfte, Von der Plünderung fremder Kulturen ». Dieses Werk unterstreicht die Rolle der Schweiz als Drehscheibe imKunsthandel.

1992 - 1993 Parlamentarische Vorstösse

- Motion von Nationalrätin Grossenbacher vom 18.6.1992

Diese Motion geht von einer bedeutenden Rolle der Schweiz «als Umschlagplatz für illegale Geschäfte mit Kunst und Kulturgütern » aus. Sie verlangt vom Bundesrat gesetzliche Massnahmen, um diesen entgegenzuwirken, die Ratifikation der UNESCO-Konvention von 1970 und den Erlass eines Einführungsgesetzes, kantonale Aufklärungskampagnen und eine Abstimmung des Schweizer Rechts auf die EG-Regelungen.

Der Nationalrat hat die Motion am 25. Juni 1993 angenommen. Sie geht nun an den Ständerat.

- Postulate der Kommissionen für Wissenschaft, Bildung und Kultur des National- und des Ständerats, 1993

Die Kommissionen für Wissenschaft, Bildung und Kultur des Nationalrats (22.1.1993) und des Ständerats (4.5.1993) forderten den Bundesrat im Zuge der Beratung des Kulturförderungsartikels mit je einem gleichlautenden Postulat auf, umgehend die UNESCO-Konvention von $1970 \mathrm{zu}$ unterzeichnen und die rechtlichen Bestimmungen zu erlassen, um den Verlust von nationalem Kulturgut zu verhindern und ausländische Staaten bei der Wahrung ihres kulturellen Erbes zu unterstützen.

Beide Vorstösse wurden in der Frühjahrs- bzw. in der Sommersession 1993 überwiesen.

- Motion von Nationalrat Keller vom 4.3.1993 Dieser Vorstoss betrifft einen Teilaspekt des Kulturgütertransits, indem er die Unterstützung von nationalen und internationalen Bestrebungen zur Zusammenführung von Kulturgütern fordert. Er wurde in der Sommersession 1993 in Form eines Postulats überwiesen.

September 1993 Vernehmlassungsverfahren zur Ratifikation der UNESCO-Konvention von 1970 und über die Einführung einer Gesetzgebungskompetenz des Bundes im internationalen Kulturgütertransfer.

\begin{tabular}{ll}
\hline Januar 1996 & $\begin{array}{l}\text { Veröffentlichung der Ergebnisse der im September 1993 eröffneten Ver- } \\
\text { nehmlassung. }\end{array}$ \\
\hline Januar 1996 & $\begin{array}{l}\text { Vernehmlassungsverfahren zur Unidroit-Konvention vom 24. Juni 1995 } \\
\text { über gestohlene oder rechtswidrig ausgeführte Kulturgüter. }\end{array}$ \\
\hline Mai 1996 & $\begin{array}{l}\text { Veröffentlichung der Ergebnisse der im Januar 1996 eröffneten Vernehm- } \\
\text { lassung. }\end{array}$ \\
\hline 30. Juni 1996 & $\begin{array}{l}\text { Unterzeichnung der Unidroit-Konvention durch die Schweiz (Beschluss } \\
\text { des Bundesrates). }\end{array}$ \\
\hline
\end{tabular}




\section{QUELLEN}

Offizielle Dokumente

- UNESCO-Konvention von 1970 über Massnahmen zum Verbot und zur Verhütung der unzulässigen Einfuhr, Ausfuhr und Übereignung von Kulturgut.

- Unidroit-Konvention vom 24. Juni 1995 über gestohlene oder rechtswidrig ausgeführte Kulturgüter.

- Bericht der Arbeitsgruppe zu Handen des Bundesamtes für Kultur und des Eidgenössischen Departements des Innern,

« Kulturgüter in der Schweiz - Einfuhr, Ausfuhr, Handel. Nationale und internationale Tragweite ; Fragestellung, Lösungen und Auswirkungen. " Januar 1991.

- Texte und erläuternder Bericht des Eidgenössischen Departements des Innern, « Handel und Verkehr mit Kulturgütern », Bern, August 1993.

- Text und erläuternder Bericht des Eidgenössischen Departements des Innern, « Unidroit-Konvention », Februar 1996.

- Ergebnisse des Vernehmlassungsverfahrens zu « Handel und Verkehr mit Kulturgütern », Bundesamt für Kultur, 1995. sowie die diesbezügliche Pressemitteilung (EDI, Januar 1996).

- Ergebnisse des Vernehmlassungsverfahrens zur «Unidroit-Konvention », Bundesamt für Kultur, Mai 1996, sowie die diesbezügliche Pressemitteilung (EDI, Juni 1996).

Veröffentlichungen

- La libre circulation des collections d'objets d'art, Quentin Byrne-Sutter et Marc-André Renold, Centre du droit de l'art, éd. Stämpfli, Zurich, 1993.

- Uniform Law Review - Revue de droit uniforme 1996-1, « Une avancée du droit international : la Convention de Rome d'Unidroit sur les biens culturels volés ou illicitement exportés ", Pierre Lalive d'Epinay.

- Götter, Gräber und Geschäfte, Von der Plünderung fremder Kulturen, Elisa Fuchs, Erklärung von Bern, April 1992.

Presseartikel

- EvB Dokumentation, 2/1992, Geplünderte Kulturen - grosse Geschäfte

- Vers un développement solidaire, Le marché de l'art, n 113, Juli 1992.

- Le Courrier, 19. Februar 1994, La liberté du commerce de l'art suscite une très vive controverse.

- NZZ, 14. Januar 1994, Umstrittene Handelsvorschriften für Kulturgüter.

- Aufzeichnung der Sendung « Forum » des Welsschweizer Radios, 16. Mai 1994, Le marché de l'art en Suisse.

- La liberté, 18. Juli 1995, La restitution d'oeuvres d'art volées sera réglementée.

- Journal de Genève, 26. Juni 1995, Accord sur la restitution d'oeuvres d'art volées.

- Journal de Genève, 20. November 1995, Pour les marchands d'art, la Suisse est un ilôt de liberté qu'ils comptent bien protéger.

- Journal de Genève, 18. Januar 1996, La Suisse aimerait mieux protéger les biens culturels étrangers.

- NZZ, 18. Januar 1996, Dubiosen Geschäften mit Kulturgütern einen Riegel schieben.

- NZZ, Unidroit-Konvention und schweizerisches Recht.

- Journal de Genève, 3. Mai 1996, Beilage Le marché de l'art.

- Journal de Genève, 7. Juni 1996, Réglementer le marché de l'art, le Conseil fédéral doit bientôt trancher.

- NZZ, 15. Juni 1996, Unidroit - Kulturgüterrecht auf Irrwegen.

- NZZ, 19. Juni 1996, Unterzeichnung der Kulturgüterschutzkonvention.

INTERNET-ADRESSE :

http ://www.access.ch/evb

Dossier zum Thema der Erklärung von Bern. Der illegale Kulturgüterhandel und die Rolle der Schweiz

\section{NUTZLICH ADRESSE :}

Centre de droit de l'art, Case postale 176, 1211 Genève 12 